\title{
Impact of Social Media on Mental Health: A Review of Cases
}

\author{
Tina Solomons \\ General Sir John Kotelawala Defence University (KDU), Ratmalana, Sri Lanka
}

\begin{abstract}
Use of social media has become a much discussed topic in the current world. Yet there is a scarcity of research literature on the impact it makes on human lives. The impact of social media on mental health has been an important topic worldwide. Sri Lanka as a country has an ever increasing social media presence at present. While some researchers argue that there are significant negative effects of social media on mental health, current research is inconclusive on this. The current paper discusses how the use of social media creates a strong impact on the psychological condition of patients who attend psychotherapy. This impact could be both positive and negative. The paper further observes how its strong influence can be felt as precipitating, perpetuating and protective factors in the course of mental illnesses, with reference to 5 cases. The paper emphasizes the inclusion of social media impact as part of case formulation and in the design of psychotherapeutic interventions. Further it emphasizes the requirement of an individual case formulation before deciding upon whether the influence of social media on the patient is negative or positive as the impact of the social media appears to interact with individual cognitive and personality factors.
\end{abstract}

Keywords: Social media, Mental Health, Psychotherapy, Case formulation

\section{Introduction}

Author is a registered clinical psychologist, who provides consultations both in the public and private sector. The author has come across many patients whose mental health was significantly affected by the use of social media, both positively and negatively. While there are many positive impacts of the current use of social media, it is important to identify it as a potential risk factor for mental health. Yet the current research literature is inconclusive on whether the impact of social media is primarily negative or positive on mental health. (Strickland \& Amelia, 2014, Pantic \& Igor, 2014) (Bashir, Hilal, \& Shabir, 2017). Some research has suggested correlations between mental illnesses such as depression and anxiety (Hughes \& Sean, 2018). Further, recent research has also suggested that some of the claims of extreme negative consequences of social media use such as being related to violence are misplaced. Yet there is some evidence that "vaguebooking", which is posting unclear but alarming sounding posts to get attention , may predict loneliness and suicidal ideation (Berryman, Chloe, Christopher, \& Charles, 2017).

The current study reviews 5 chosen cases from the author's clinical records, which, in author's opinion, social media has had a significant impact.

\section{Methodology}

The cases were selected from the author's clinical records. The author has selected cases which demonstrated clear effects of social media on various stages of mental health difficulties, as per her subjective opinion and clinical judgement. This selection was guided by the author's clinical experience and reference to current literature on the subject.

Verbal consent is obtained from each patient when they commence treatment on the possible use of clinical records in teaching exercises and research publications that may be done in future. Further the patients are educated on their rights of withdrawal of such consent. The cases included in the current study are of patients 
who did not have any objection to such use of their data. Where necessary written permission has also been obtained. Further, their identity has been adequately concealed in the current paper, so as to protect their confidentiality

\section{Case Series}

\section{Case of " $A$ "}

"A" was a known patient of Obsessive Compulsive Disorder who has been on treatment with pharmacotherapy and psychotherapy for close to 7 years. The patient self-referred to the author who is a registered clinical psychologist. The patient presented in the private sector for consultations in a well-known private hospital. The patient has been on psychotherapy with few different therapists and few different psychiatrists intermittently. She acknowledged that most of these treatments had helped her in the past. When she presented to the author she was in a relapse, with acute symptoms. Her psychiatrist had already increased the dosage of her drug regime. She was treated with an SSRI (Selective Serotonin Reuptake Inhibitors)

"A" was 26 years old, and was educated up to postgraduate level. She was the older child in a family with two daughters. She came from an upper middle class family with stable economic standing. She had a close relationship with her family. She improved rapidly on her OCD symptoms. Her obsessions and compulsions reduced. Her prior knowledge on psychotherapeutic process and techniques, aided her rapid progress.

After the remission of the acute stage, it was apparent that the patient displayed a considerable amount of trauma related symptoms. Specifically frequent feelings of emotional numbness and disconnection from emotions, strong feelings of guilt and self-blame, feelings of derealisation and confusion and were present. According to the patient she felt she could never completely recover due to these symptoms becoming prominent whenever she progressed in treatment. With further exploration the patient revealed the memory of an incident which occurred seven years ago, where one of her past romantic partners had publicised naked pictures of herself in social media. The patient has become aware of this, through her friends, and has subsequently complained to the cyber security division of Sri Lanka police, which has taken the necessary measures to remove the pictures and subsequently warn the partner. This incident which is a very severe form of emotional abuse had resulted in emotional trauma in the patient. Immediately after this, the onset of OCD had occurred in the patient.

\section{Case of $B$}

"B" was a 27 year old patient diagnosed was recently diagnosed with depression. The patient has already consulted a psychiatrist and was already on an SSRI treatment regime. When the patient started on psychotherapy, after initial progress, appeared to relapse repeatedly. Especially the feelings of hopelessness were re-fuelled by lack of likes for her pictures on Facebook (FB), and seeing the success of her rivals, who were nevertheless friends on FB. She unreasonably compared herself with her virtual network, which aggravated her depressive symptoms and counteracted the effects associated with psychotherapy. In addition to this, due to lack of motivation, FB use had become an easy distraction. This was resulting in lack of productivity in the work place, which further contributed to the depression. This was hindering her motivation try to create new habits which would make her more productive, which would in turn help her feel positive about herself. Thus, FB had become a part of the typical vicious cycle seen in depression.

This patient took a short break from all social media, until she developed the skills overcome her depressive symptoms. When the depressive symptoms were in remission, she was mastered the cognitive skills required to help her respond to social media appropriately. This, part of the treatment was specifically important and helpful in preventing relapse as was revealed by the patient in booster sessions conducted after the termination of psychotherapy. 


\section{Case of $C$}

"C" was a 38 year old patient Diagnosed with paranoid schizophrenia. Her symptoms were in remission and had well-adjusted a life style of a house wife who could adequately take the responsibilities of her household work and caring for her child. She was also compliant with the treatment regime with a psychiatrist and followed advice on preventing relapse. She was primarily treated with Second generation Antipsychotic (SGA). She had recently opened an FB account and was following advice on a women's group on infidelity of husbands. This had re-activated her paranoid ideation about her husband's potential infidelity. While she keenly re-checked her husband's FB profile several times a day, she complained that her husband who always had an FB account never encouraged her to have one because he intended on being unfaithful.

\section{Case of "D"}

D was 30 year old a female who presented with mild level depressive symptoms after a recent break up of a romantic relationship. She was rational about the breakup of her relationship. Despite this, she was developing depressive symptoms. After the initial few sessions she was advised to keep a diary on her mood. This is a standard exercise in Cognitive Behaviour Therapy to identify the triggers of emotional fluctuations. This revealed that thoughts about the current actions of her former partner were the most common reasons for ongoing sadness and anger, which were rated from $60 \%-80 \%$. On further exploration the patient realised that most of these thoughts resulted after seeing the pictures on the ex-partner shared on FB. In addition to that, posts and messages which he shared on common groups in social media such as Viber, WatsApp etc were also affecting her. Upon this realization the patient decided to take a short break from all social media. She described that constant encounters with her ex-partner on social media somehow made her feel as if the break out was not real and lead her to react to him as if she was still in a relationship with her. In her own words, she wanted to have a real break from the person to feel the break up was real and that required disconnecting from virtual reality.

After she was sufficiently stable in her emotions she resumed the use of social media after learning emotional and cognitive skills necessary for using social media in a way that did not negatively affect her psychological condition. Further, the patient suggested that taking a break from social media must be just like going on vacation, something people should do on a regular basis to refresh and rejuvenate.

\section{Case of $E$}

"E" was a patient who was recovering from a primary depression and a secondary anxiety. While her depressive symptoms and anxiety symptoms were in remission, she still struggled at finding a way of income to support her. Her dependency on her parents at the age of 28 bothered her and thoughts associated to this were triggering depressive mood. After discussions on identifying her strengths, she identified that she is competent in making greeting cards which are of sellable quality. Yet she felt that she lacked the resources to connect with sellers. After further consideration, she decided to directly attempt at selling her cards through an FB page. This succeeded steadily and was helping the patient develop more rational and positive thoughts about her strengths. Further, appreciative comments from others on her creations had a positive impact on her self-esteem. During the course of therapy she also learnt skills to respond to possible negative comments in future. 


\section{Case of $F$}

"F" was a 28 year old man who wanted to get in to a romantic relationship. According to him each time he tried to talk to a potential romantic partner, he was feeling a bit anxious. Yet he did not fulfil criteria for any diagnosis. Therefore the consultations were more directed at discussions about the aspects of communication skills he should practice and relationship goals and expectations he has. While on therapy he registered on an online dating site and started talking to a potential partner. He applied some of the communication skills he learnt in therapy in the virtual chat environment. According to the patient he felt comfortable to call the person after chatting in the virtual environment. After that, he proceeded to call and finally meet. Though there was some amount of common anxiety that was involved in meeting a romantic partner for the first time, the patient reported that he was able to manage the meeting and start dating the person with a view to gauging their suitability as long term partners.

\section{Discussion and implications for clinicians}

According the experience of the author the majority of the patient population who are below 55, from urban areas are active on social media. It serves as a mode of information sharing and networking. When the gender differences are compared, women appear to interact on social media on a more personal basis where there are more personal emotions are shared openly with a group, whereas men appear to share more of opinions, political ideologies and humour.

When the case series of the current paper is analysed, it is clear that individual responses to social media and its impact on their mental health also depends on individual factors. In addition to that it is noteworthy for all mental health professionals to note that the interaction between mental illnesses and social media should always be taken into account in providing treatment. When the case "A" is considered it appears that that the social media influence and the subsequent abuse has acted possibly as precipitating factor for the mental illness. This influence appeared to have interacted with the patient's already existing anxious personality type, genetic and biological make up and other stressors that were present. Thus it can be speculated that the social media influence would have interacted with the predisposing factors that were already present in the patient.

In case "B", social media influence would have acted as a precipitating factor as in case "A". Yet what is prominent is its influence as a maintaining or in other words a perpetuating factor in patient's condition. The author has observed this perpetuating influence in numerous other patients who has not been discussed in this paper.

In case "C" it appeared to have acted as a trigger for relapse. Her paranoid ideation had been triggered by the discussions on the women's group on infidelity of husbands. This, if it was left not being discussed in the treatment, would have led to a full blown psychotic relapse in the patients. Therefore the influence of social media can also act as a trigger for relapse and thereby influencing the course of the illness.

In case " $\mathrm{D}$ " the person again it appears to have acted as a perpetuating factor for the patient's relationships issues and her depression. It would have also played a part in the onset of the depression, interacting with the stress of the break up and other predisposing factors that would have been present in the patient.

In the case of "E" it can be seen as factor that supported the patient recover. In addition to that it prevented the patient from relapsing again due to his financial dependency on the family. Therefore it could also be seen as a protective factor. The same influence was seen in the case " $\mathrm{F}$ ", where the chance for the patient to practice his newly learnt communication skills in the virtual environment and build his self-esteem in the area of communication acted as a protective factor against developing further difficulties. This is specially so, as exposure to a negative experience of interaction with others can create fear and anxiety about subsequent 
interactions. Therefore this may have protected the person from feelings of loneliness which could lead to depressive symptoms and anxiety related symptoms.

\section{Conclusions}

It is clear that in the current environment the influence of social media should be considered at all stages of mental illness. It is recommended that it be included as component of case formulation at all stages. This would allow clinicians to intervene these influences if necessary at all stages. Further, the popular opinion which is skewed toward social medial always acting as a negative influence should not be generalized to all cases, as it can also act as a protective factor. Therefore, to better understand nature and extent of the influence of social media in a person, an individual case formulation should be carried out, taking into consideration the individual factors.

As this is based only on clinical experience and clinical notes, it is highly recommended that future researches conduct systematic research on the influence of social media on mental health.

\section{References}

Bashir, Hilal, B., \& Shabir. (2017). Effects of Social Media on Mental Health: A Review. The International Journal of Indian Psychology, 2349-3429.

Berryman, Chloe, F., Christopher, N., \& Charles. (2017). Social Media Use and Mental Health among Young Adults. Psychiatric Quarterly.

Hughes, \& Sean. (2018). The Effects of Social Media on Depression Anxiety and Stress . Dublin: Dublin Business School.

Pantic, \& Igor. (2014). Online Social Networking and Mental Health. Cyberpsychology , Behaviour , and Social Networking, 652 - 657.

Strickland, \& Amelia. (2014). Exploring the Effects of Social Media Use on the Mental Health of Young Adults. Orlando: University of Central Florida. 